\title{
Study of the Growth Control of Nonionic Surfactant Reverse Micelles by Water and Glycerol Using Small- angle X-ray Scattering
}

\author{
Lok Kumar Shrestha* \\ Graduate School of Environment and Information Sciences, Yokohama National University, \\ Tokiwadai 79-7, Hodogaya-ku, Yokohama 240-8501, Japan. \\ e-mail:lokkumar@hotmail.com
}

\begin{abstract}
This paper described a facile route to the growth control of nonionic surfactant reverse micelles in diglycerol monolaurate $\left(C_{12} G_{2}\right) /$ cyclohexane systems at $25^{\circ} C$. Structural characterization of reverse micelles was carried out using small-angle $X$-ray scattering (SAXS) technique. It has found that $C_{12} G_{2}$ spontaneously formed elongated prolate type reverse micelles in cyclohexane at $25^{\circ} \mathrm{C}$ and the micellar size grows with $\mathrm{C}_{12} \mathrm{G}_{2}$ concentration. The reverse micelles could solubilize some amount of water in the micellar core thus enhanced their potential application in the encapsulation of water-soluble drugs. Phase separation occurred with further addition of water. The micelles grow with the amount of water content in the system, as water tends to go to the micellar core swelling the micelles. However, the size decreased with increasing temperature at fixed water content. Increasing mixing fraction of glycerol in water decreased the micellar size. This would be understood as glycerol dehydrates surfactant's headgroup and increased the packing parameter.
\end{abstract}

Keywords: Diglycerol monolaurate, cyclohexane, glycerol, small-angle X-ray scattering (SAXS), reverse micelles.

\section{Introduction}

Reverse micelles have an inverted structure compared to the conventional normal micelles in aqueous systems. Therefore, it is often known as inverse or inverted micelles. The micellar cores consist of a hydrophilic polar component and the shells consist of a lipophilic nonpolar part of the surfactant molecules in the reverse micelles. The dipole-dipole interaction between the hydrophilic head groups is assumed to be one of the driving forces for the formation of reverse micelles in organic solvents. ${ }^{1}$ Studies on the formulation of reverse micelles and their structure control have attracted a significant interest over the years

"Corresponding author 
in surfactant sciences due to its wide range of applications. Reverse micelles have been used as relevant templates for the synthesis of nanomaterials and also as a size controlling microreactor for various aqueous chemical reactions. ${ }^{2-4}$ The ternary mixtures of water/sodium bis(2-ethylhexyl) sulfosuccinate (AOT)/oil or water/lecithin/oil have mostly been considered for the studies of reverse micelles or water in oil (W/O) microemulsions so far ${ }^{5-10}$ However, the structure of reverse micelles in nonionic surfactant/oil systems is less frequently studied. Glycerol-based nonionic surfactants have attracted considerable attention from cosmetic and food industries. These surfactants are derived from natural sources and are biodegradable and biocompatible. Thus, the formulation of reverse micelles and their free structure control by solubilizing polar components in their micellar core would offer various practical applications. In this context, the present work is an extension of the previously published work in which the structure of the nonionic surfactant diglycerol monomyristate $\left(\mathrm{C}_{14} \mathrm{G}_{2}\right)$ micelles in cyclohexane $(\mathrm{CH})$ as a function of surfactant concentration, temperature and water was investigated. ${ }^{11}$

The present work is aimed to study the SAXS effects of surfactant concentration, added water, and mixing fraction of glycerol (Gly) in water on the reverse micelles structure of diglycerol monolaurate $\left(\mathrm{C}_{12} \mathrm{G}_{2}\right)$ surfactant.

\section{Experimental Methods}

The nonionic surfactant $\mathrm{C}_{12} \mathrm{G}_{2}$ was obtained from Taiyo Kagaku Co., Ltd., Yokkaichi, Japan. The surfactant was $91.1 \%$ pure and the main impurities present were unreacted diglycerol, diglycerol difatty acid esters. The surfactant was used as received without further purification. Cyclohexane $(\mathrm{CH})$ was purchased from Tokyo Chemical Industry, Tokyo, Japan. Commercially available glycerol (Gly) and Millipore water was used as polar additives.

5 and $10 \mathrm{wt} \%$ of $\mathrm{C}_{12} \mathrm{G}_{2}$ solutions were prepared in $\mathrm{CH}$, and taken in clean and dry glass ampoules with screw cap. The samples were mixed properly by using dry thermo-bath, vortex mixer and repeated centrifugation to achieve homogeneity. Following similar method, ternary mixtures of $\mathrm{C}_{12} \mathrm{G}_{2} / \mathrm{CH} /$ water with water composition $0,0.4,0.8$ and 1.0 $\mathrm{wt} \%$ in $10 \mathrm{wt} \% \mathrm{C}_{12} \mathrm{G}_{2} / \mathrm{CH}$ were prepared. Similarly, $10 \mathrm{wt} \% \mathrm{C}_{12} \mathrm{G}_{2} / \mathrm{CH}+1.0 \%$ Gly:water $=$ 0:100, 20:80, 40:60, and 60:40 solutions were prepared. All the samples were placed in a temperature-controlled water bath at $25^{\circ} \mathrm{C}$ for few hours before SAXS measurements.

A SAXSess camera (Anton Paar, Austia) attached to a PW3830 laboratory X-ray generator with a long fine focus sealed glass X-ray tube $\left(K_{\alpha}\right.$ wavelength of $\left.0.1542 \mathrm{~nm}\right)$ was used. The apparatus was operated at $40 \mathrm{kV}$ and $50 \mathrm{~mA}$. An equipped Göbel mirror and a block collimator enabled us to obtain a focused monochromatic X-ray beam of $\mathrm{Cu} \mathrm{K}$ radiation $(\lambda=0.1542 \mathrm{~nm})$ with a well-defined line-shape. A thermostated sample holder unit was used to control the sample temperature. The $2 \mathrm{D}$ scattering pattern was recorded by an imaging-plate and integrated into to one-dimensional scattered intensities $I(q)$ as a function of the magnitude of the scattering vector $q=(4 \pi / \lambda) \sin (\theta / 2)$ using SAXSQuant software (Anton Paar), where $\theta$ is the total scattering angle. All measured intensities were semiautomatically calibrated for transmission by normalizing a zero- $q$ attenuated primary 
intensity to unity, by taking advantage of a semi-transparent beam stop. All $I(q)$ data were corrected for the background scattering from the capillary and the solvents. Furthermore, the absolute scale calibration was made using water as a secondary standard. To obtain realspace structural information, SAXS data were evaluated by generalized indirect Fourier transformation (GIFT) method. ${ }^{12.13}$ Averaged structure factor of hard-sphere interaction potential model, ${ }^{14,15}$ and Percus-Yevick closure relations to solve Ornstein-Zernike equation were used. The detailed theoretical description on the method is described elsewhere. ${ }^{16,17}$

\section{Results and Discussion}

\section{Effect of Surfactant Concentration on the Reverse Micellar Structure:}

Figure 1 shows the X-ray scattering intensities, $I(q)$, and the corresponding pair-distance distribution functions (PDDFs), $p(r)$, deduced from the GIFT evaluation of the SAXS data at two different concentrations of $\mathrm{C}_{12} \mathrm{G}_{2} / \mathrm{CH}$ surfactant (5 and $10 \mathrm{wt} \%$ ) at $25^{\circ} \mathrm{C}$.
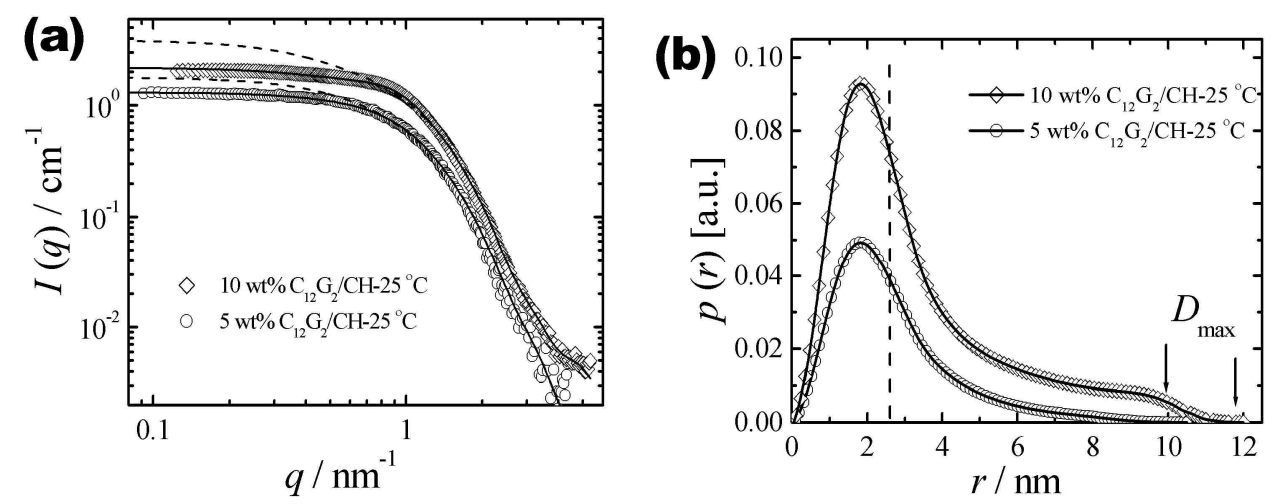

Figure 1: (a) The scattering intensities, $I(q)$, and (b) the corresponding $p(r)$-function. The solid and broken lines in panel (a) and in the subsequent figure represents the GIFT fit and the calculated form factor, $P(q)$, for $n$ particles existing in unit volume $n P(q)$. The broken line and arrows in the higher-r side of the panel $(b)$ and in the subsequent figure highlights the cross-sectional diameter and the maximum dimension of the micelle.

The strong $q$ dependence of $I(q)$ in the scattering curves is a clear signature of the formation of reverse micelles in the $\mathrm{C}_{12} \mathrm{G}_{2} / \mathrm{CH}$ system. One can anticipate a negligible intermicellar interactions within the studied concentrations as the scattering curves do not exhibit any correlation peak in the low- $q$ regime of the $I(q)-q$ curve. With increasing concentration, the scattering intensity increases throughout the entire $q$-range and even if one normalizes the scattering intensity by volume fraction of the surfactant, the scattering at higher concentration will be higher. This shows the micellar growth induced by the surfactant concentration, which can also be seen in $p(r)$-functions as shown in Fig. 1(b). The $p(r)$ curves exhibit typical feature of elongated ellipsoid prolate type micelles judged from the pronounced peak in the low- $r$ side with an extended downward convex tail to the higher- $r$ side. ${ }^{18,19}$ With increasing $\mathrm{C}_{12} \mathrm{G}_{2}$ concentration from 5-10 wt $\%$, the maximum dimension of 
the micelles $\left(D_{\max }\right)$ was increased from $\sim 10$ to $12 \mathrm{~nm}$ keeping the inflection point seen after the maximum of the $p(r)$-functions in the low- $r$ side (as indicated by a broken line) constant. This confirmed that the local internal structure, i.e., the cross-sectional diameter of the micelles remained essentially the same despite the change in surfactant concentration. The micellar growth induced by surfactant concentration can be explained in terms of critical packing parameter ( $c p p)$. In general, the $c p p$ tends to decrease with increasing surfactant concentration due to packing constraints. The decreased in the $c p p$ in reverse system favored the micellar growth.

\section{Effect of Added Water on the Reverse Micellar Structure:}

Figure 2 shows the $I(q)$ and corresponding $p(r)$-curves for the $10 \mathrm{wt} \% \mathrm{C}_{12} \mathrm{G}_{2} / \mathrm{CH}+$ water systems at different concentrations of water $(0,0.4,0.8$, and $1.0 \%)$ at $25^{\circ} \mathrm{C}$. As can be seen in Fig. 2(a), the scattering intensity in the low- $q$ region is strongly enhanced and the scattering curve in the high- $q$ region (about $2 \mathrm{~nm}^{-1}$ as indicated by an arrow) shifts towards the forward direction upon addition of water giving a clear indication of water induced micellar growth. The corresponding real space $p(r)$-functions revealed that addition of water induced dramatic changes in the structure of the reverse micelles in terms of maximum dimensions $\left(D_{\max }\right)$ and the internal cross-sectional structure as shown in Fig. 2(b). Furthermore, addition of $0.4 \%$ water to the $10 \mathrm{wt} \% \mathrm{C}_{12} \mathrm{G}_{2} / \mathrm{CH}$ system increased $D_{\max }$ from 12 to $14 \mathrm{~nm}$. Besides, the cross-sectional diameter as indicated by broken lines after the maximum of the $p(r)$-curves shifted towards higher- $r$ side. These features were also discussed in water induced micellar growth. ${ }^{20,21}$ Minute observations of the $p(r)$-curves revealed that water not only changes the size but also the shape of the micelles. The shape of the $p(r)$-curves of water content systems, i.e., the pronounced peak in the low- $r$ side with an extended long tail in the higher-r side, is close to that of the rod-like micelles. When water is added in the reverse micellar solution, it tends to form water pool in the micellar core, as water is less miscible with organic solvent. Furthermore, there is a possibility of hydrogen bond formation between water and the glycerol molecule, which increased the headgoup size of the surfactant and favored micellar growth by decreasing срp.
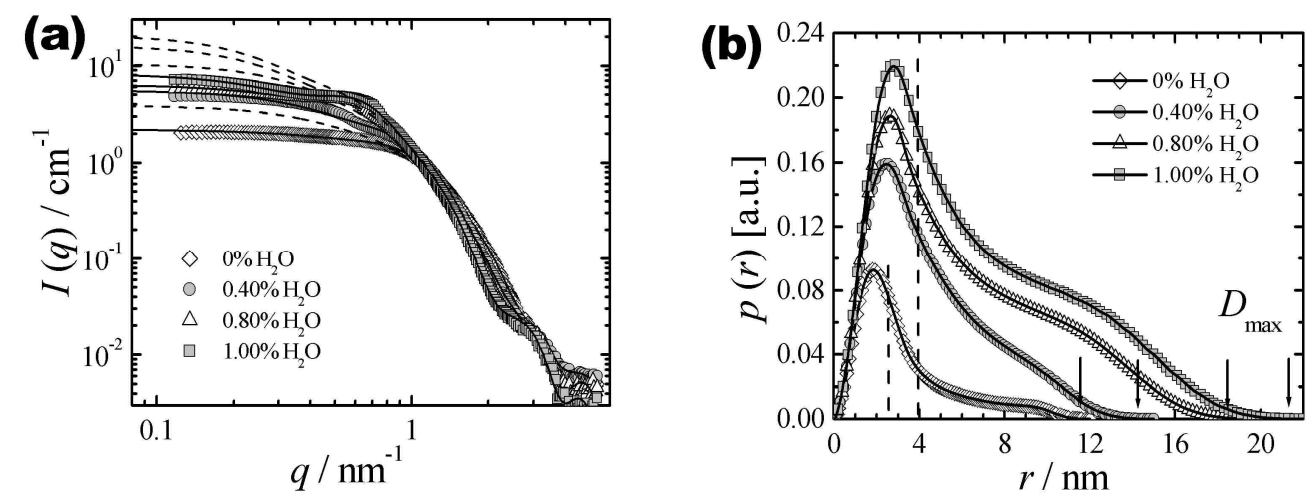

Figure 2: (a) The I(q) of $10 \mathrm{wt} \% \mathrm{C}_{12} \mathrm{G}_{2} / \mathrm{CH}+$ water systems as a function of water content at $25^{\circ} \mathrm{C}$ and (b) the corresponding $p(r)$-curves. 
Figure 3 shows the result of temperature effect on reverse micellar structure of $10 \mathrm{wt} \%$ $\mathrm{C}_{12} \mathrm{G}_{2} / \mathrm{CH}+1.0 \%$ water system. Increasing temperature decreases the scattering intensity in the low- $q$ region without affecting the scattering behavior in the high- $q$ regions as shown in Fig. 3(a). The decreasing trend of the low- $q$ scattered intensity with increasing temperature can be taken the evidence of the decreasing micellar size. ${ }^{20}$ The real space $p(r)$-function showed that the size of the micelles decreased from $\sim 22$ to $12 \mathrm{~nm}$ upon increasing temperature from 25 to $55^{\circ} \mathrm{C}$. However, the cross-sectional diameter remains unchanged $(\sim$ $4 \mathrm{~nm}$ ) as shown in Fig. 3(b). Judging from the trend of shape and size changes with temperature, it is possible to anticipate the formation of globular type of reverse micelles at sufficient higher temperature. Thus, one can conclude that the length of the aggregates decreased with the rise of temperature, which is essentially a rod-to-sphere type transition in the reverse micellar structure. The structural transition could be understood in terms of critical packing. Increasing temperature dehydrated the hydrophilic headgroup of the surfactant and thus by increasing cpp decreased the micellar size.
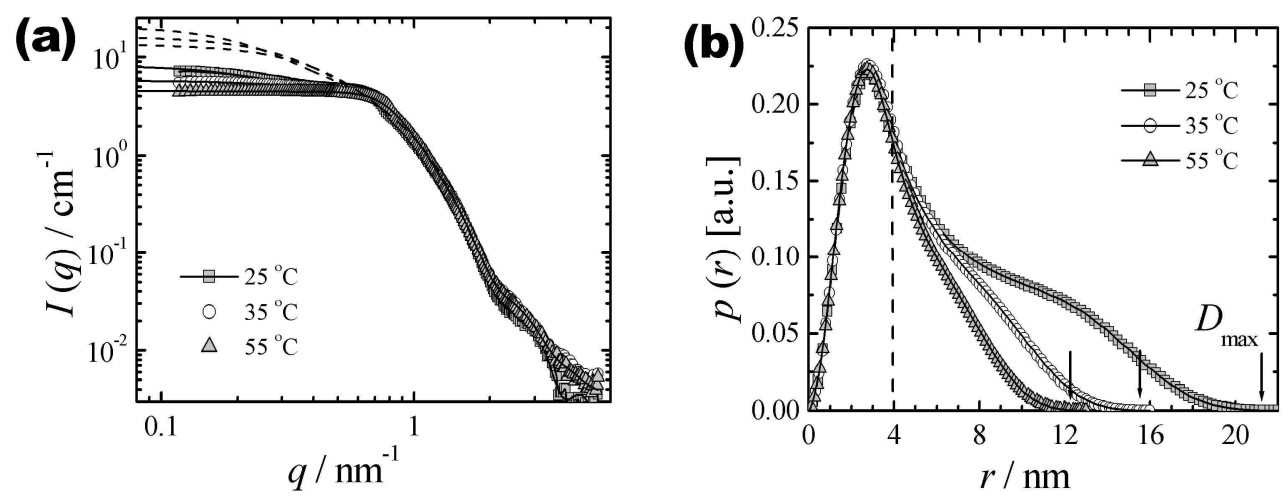

Figure 3: (a) The I(q), of the $10 \mathrm{wt} \% \mathrm{C}_{14} \mathrm{G}_{2} / \mathrm{CH}+1 \%$ water system at different temperatures and $(b)$ the corresponding $p(r)$-functions.

\section{Effect of Mixing Fraction of Glycerol in Water on the Reverse Micellar Structure:}

Figure 4 shows the effect of mixing fraction of glycerol in water on the $I(q)$ and $p(r)$ functions of the reverse micelles of $10 \mathrm{wt} \% \mathrm{C}_{12} \mathrm{G}_{2} / \mathrm{CH}+1 \%$ Gly:water system at $25^{\circ} \mathrm{C}$. Increasing mixing fractions of glycerol in water decreases the low- $q$ scattering intensity. However, the scattering functions in the higher- $q$ region $\left(q \sim 2 \mathrm{~nm}^{-1}\right)$ tend to shifted towards higher- $q$ regions. These features show that increasing mixing glycerol in the system reduced the micellar size, which is evident in the $p(r)$-curves as shown in Fig. 4 (b). With increasing mixing fractions of glycerol from Gly:water $=0: 100$ to Gly: water $=60: 40$, the micellar size decreased from $\sim 22$ to $16 \mathrm{~nm}$. Besides, the micellar cross-section decreases from $\sim 4$ to 3 $\mathrm{nm}$. The structural transition induced by glycerol can be understood in terms of the fact that glycerol dehydrates the surfactant's headgoup, which is hydrated by water. This leads to decreases in the effective area of hydrophilic headgroup and thus increased the cpp. As a result, aggregates with more negative curvature would like to form. 

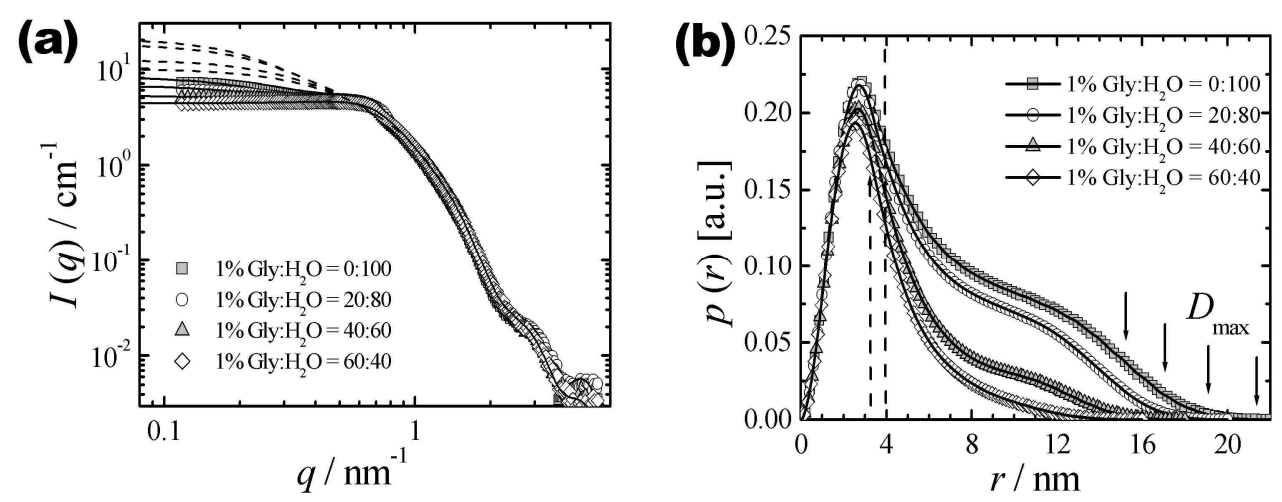

Figure 4: (a) The I(q), of the $10 \mathrm{wt} \% \mathrm{C}_{14} \mathrm{G}_{2} / \mathrm{CH}+1 \%$ Gly: $\mathrm{H}_{2} \mathrm{O}$ systems at different mixing fractions of Gly in $\mathrm{H}_{2} \mathrm{O}$ and $(b)$ the corresponding $p(r)$-functions.

\section{Conclusions}

The paper has shown the facile routes to the growth control of nonionic surfactant reverse micelles by surfactant concentration, water and glycerol. Depending on the surfactant concentration, the micellar size differed showing the formation of longer micelles at higher concentrations. The reverse micellar size increased continuously with water content favoring the formation of long rod-like micelles at higher water content. At fixed water content, the micellar size decreased with the rise of temperature. Similarly, increasing mixing fraction of glycerol in water decreased both the maximum dimension, and crosssection of the micelles. The present investigation has clearly shown the possibility to the structural control of reverse micelles and thus offered fundamental and practical implications.

\section{Acknowledgements}

A thanks JSPS for the research grant, and Prof. Kenji Aramaki, Yokohama National University for the laboratory facilities. Technical assistance of Dr. Rekha Goswami Shrestha, Tokyo University of Science is highly acknowledged.

\section{References}

1. J. N. Israelachvili, D. J. Mitchell, B. W. Ninham, J. Chem. Soc. Faraday Trans.2, 1976, $72,1525$.

2. P. L. Luisi, B. E. Strab (Eds.) Reverse Micelles: Biological and Technological relevance of Amphiphilc Structures in Apolar Media; Plenum Press: New York, 1987.

3. M. A. López-Quintela, C. Tojo, M. C. Blanco, L. García Rio, J. R. Leis, Current Opinion in Colloid and Interface Science 2004, 9, 264. 
4. I. Lisiecki, M. P. Pileni, J. Am. Chem. Soc. 1993, 115, 3887.

5. R. E. Riter, J. R. Kimmel, E. P. Undiks, N. E. Levinger, J. Phys. Chem. B., 1997, 101, 8292.

6. M. Kanamaru, Y. Einaga, Polymer, 2002, 43, 3925.

7. J. P. Cason, C. B. Roberts, J. Phys. Chem. B., 2000, 104, 1217.

8. Q. Li, T. Li, J. Wu, J. Phys. Chem. B., 2000, 104, 9011.

9. T. De, A. Maitra, Adv. Colloid Interface Sci. 1995, 59, 95.

10. S-H. Tung, Y-E. Huang, S. R. Raghavan, J. Am. Chem. Soc. 2006, 128, 5751.

11. L. K. Shrestha, J. Nepal Chem. Soc., 2008/2009, 23, 74.

12. G. Fritz, A. Bergmann, O. Glatter, J. Chem. Phys. 2000, 113, 9733.

13. O. Glatter, G. Fritz, H. Lindner. P.J. Brunner, R. Mittelbach, R. Strey, S.U. Egelhaaf, Langmuir 2000, 16, 8692.

14. P. N. Pusey, H. M. Fijnaut, A. Vrij, J. Chem. Phys. 1982, 77, 4270.

15. P. Salgi R. Rajagopolan, Adv. Colloid Interface Sci. 1993, 43, 169.

16. O. Glatter, Progr. Colloid Polym. Sci. 1991, 84, 46.

17. O. Glatter, J. Appl. Cryst. 1981, 14, 101.

18. L. K. Shrestha, T. Sato, K. Aramaki, J. Phys. Chem. B. 2007, 111, 1664.

19. L. K. Shrestha, T. Sato, K. Aramaki, Phys. Chem. Chem. Phys. 2009, 11, 4251.

20. L. K. Shrestha, O. Glatter, K. Aramaki, J. Phys. Chem. B. 2009, 113, 6290.

21. L. K. Shrestha, R. G. Shrestha, K. Oyama, M. Matsuzawa, K. Aramaki, J. Phys. Chem. B. 2009, 113, 12669. 